\title{
Injurias traumáticas dentales en niños y adolescentes de un Centro Dental Docente de la Universidad Peruana Cayetano Heredia
}

Traumatic dental injuries in children and adolescents of a Teaching Dental Center of the Peruvian University Cayetano Heredia

\author{
Sandra L. Alvarez-Raico ${ }^{1, a}$, Roberto A. León-Manco 2,b, María Elena Díaz-Pizán 3,c \\ RESUMEN
}

Objetivo: Determinar la frecuencia de injurias traumáticas dentales según la clasificación de Andreasen en niños de 1 a 15 años de edad, atendidos en el Servicio de Odontopediatría del Centro Dental Docente de la Universidad Peruana Cayetano Heredia, en el periodo enero 2012 a octubre 2017. Material y métodos: El estudio fue de tipo descriptivo retrospectivo y transversal. La muestra estuvo conformada por historias clínicas de pacientes con injurias traumáticas dentales que cumplieron con los criterios de inclusión y exclusión. Las variables evaluadas fueron: tipos de injurias traumáticas dentales, sexo, edad del paciente, lugar, causa del trauma y tipo de pieza dental afectada. Los resultados fueron presentados en frecuencias absolutas y relativas mediante tablas descriptivas de doble entrada. Resultados: se encontró un total de 9880 historias clínicas, de las cuales el 1,18\% $(\mathrm{n}=117)$ fueron de pacientes que presentaron injurias traumáticas dentales, con una edad media de 4,36 $\pm 3,42$ años. Conclusiones: Las injurias traumáticas dentales más frecuentes fueron las lesiones periodontales seguidas de las lesiones de la encía y/o mucosa. Los niños fueron los más afectados en la dentición primaria y las niñas en la dentición permanente. La mayoría de las lesiones traumáticas dentales se produjeron durante los juegos. La casa y el colegio fueron los lugares donde se produjo la mayor parte de los traumatismos en la dentición primaria y permanente, respectivamente. Los incisivos centrales superiores fueron las piezas dentales más afectadas en este grupo de niños.

PALABRAS CLAVE: niño, preescolar, diente primario, traumatismos de los dientes, dentición permanente.

Odontopediatría-Postgrado, Facultad de Estomatología, Universidad Peruana Cayetano Heredia. Lima, Perú.

Departamento Académico de Odontología Social, Facultad de Estomatología, Universidad Peruana Cayetano Heredia. Lima, Perú.

Departamento Académico Estomatológico del niño y del Adolescente, Facultad de Estomatología, Universidad Peruana Cayetano Heredia. Lima, Perú

Especialista en Odontopediatría.

Especialista en Salud Pública Estomatológica y Magister en Salud Pública.

Magister en Estomatología y Doctora en Estomatología. 


\section{ABSTRACT}

Objectives: To determine the frequency of traumatic dental injuries according to Andreasen classification in children from 1 to 15 years old, treated in the Pediatric Dentistry Service of the Peruvian University Cayetano Heredia Teaching Dental Center, from January 2012 through October 2017. Material and methods: This study was descriptive and retrospective. Samples were composed of clinical records from patients with traumatic dental injuries who accomplished the inclusion and exclusion criteria. The evaluated variables were: types of traumatic dental injuries, sex, age of the patient, place, cause of the trauma and type of teeth affects. Results: A total of 9880 medical records were found, of which $1.18 \%(n=117)$ represent patients who presented traumatic dental injuries. From these, 73 were from boys and 31 from girls, who had an average age of $4.36 \pm 3.42$ years old. Conclusions: Injuries to the periodontal tissue and injures to gingiva and/or mucosa were the most common injuries on both dentitions. Games were the most frequent cause for traumatic dental injuries, home was the most frequent place for primary dentition and school for the permanent dentition. Two year-old patients were the most affected in primary dentition and seven year-old patients in permament dentition.

KEY WORDS: child, preschool, tooth deciduous, tooth injuries, dentition permanent.

\section{INTRODUCCIÓN}

Las injurias traumáticas dentales vienen incrementando su prevalencia en las diferentes partes del mundo, muchos autores hacen referencia a un aumento en las niñas en los últimos años debido a su mayor participación en juegos y deportes de aventura que antiguamente eran realizados con mayor preferencia por los niños $(1,2,3,4)$. Las injurias traumáticas son relativamente comunes en los niños (Organización Mundial de la Salud 2019) (5); estudios a nivel mundial $(6,7,8,9,10,11,12,13,14,15)$ muestran que la prevalencia de injurias traumáticas dentales varía según el grupo estudiado. El meta-análisis realizado por Petti et al., señala una prevalencia de $22,7 \%$ en dientes primarios en un total de 59436 niños con una edad media de 3,4 años; siendo la prevalencia de $27 \%$ en la región de Asia Sudoriental y de 14,2\% en la región de Europa (6). En cuanto a la dentición permanente estos autores encontraron, en niños de 12 \pm 1 años una prevalencia de $18,1 \%$ de un total de 33 829 niños, encontrándose en América una prevalencia de $22.1 \%$, mientras que en África 13,2\%. Al-Bajjali y Rajab señalan que la prevalencia de traumatismos en dientes anteriores permanentes varía de $4,1 \%$ a $58,6 \%$ en el mundo (7); encontrándose en Asia valores que oscilan entre $4 \%$ y $35 \%$ y en el Medio Oriente 7,4\% (8). Los deportes, caídas y colisiones son las causas más frecuentes de las injurias traumáticas dentales; la etiología puede variar según diferentes factores como sexo, edad, medio ambiente, estatus social $(3,4,7,14)$.
Según Ogordi et al., el lugar más frecuente para traumatismos dentales es el entorno escolar seguido del hogar (16).

Estas injurias traumáticas dentales ocasionan un impacto negativo en la calidad de vida de la persona afectada $(1,2,9,12,14)$; además puede provocar dolor, infección, dificultad para hablar, restricciones para morder, pérdida dental irreversible, impacto psicológico y emocional y alteración en la apariencia física $(3,4,7,8,10,11,12,13)$.

Los niños pequeños, sobre todo los infantes, no tienen suficiente coordinación motora y conocimiento del peligro, por lo que se encuentran expuestos a las caídas dando lugar a sucesos traumáticos en diferentes grados de intensidad, afectando estructuras anatómicas bucales. La severidad del traumatismo está determinada por la fuerza y la dirección del impacto; además, existen otros factores de riesgo como la anatomía y posición del diente, overjet aumentado, el estado del desarrollo radicular, incompetencia labial, obesidad infantil, los cuales pueden incrementar la severidad $(3,4,7,9,10,13,14,17,18)$.

Este trabajo tuvo como objetivo determinar el tipo de injuria traumática dental más frecuente según la clasificación de Andreasen, de acuerdo a la edad, sexo, tipo de dentición y pieza dentaria más afectada e identificar la causa y lugar de mayor frecuencia de dichas injurias en pacientes de 1 a 15 años de edad, 
atendidos en el Servicio de Odontopediatría del Centro Dental Docente de la Universidad Peruana Cayetano Heredia, en el periodo: enero 2012 a octubre 2017.

\section{MATERIAL Y MÉTODOS}

Se realizó un estudio descriptivo, retrospectivo y transversal. Historias clínicas de pacientes de 1 a 15 años de edad que fueron atendidos en el Servicio de Odontopediatría del Centro Dental Docente de la Universidad Peruana Cayetano Heredia (UPCH), en el periodo de enero 2012 a octubre 2017 fueron revisadas, de las cuales se seleccionaron aquellas que cumplieron con los criterios de selección. Los criterios de inclusión fueron: historias clínicas de traumatismos dentoalveolares completas de pacientes de 1 a 15 años de edad, de ambos sexos que llegaron al servicio de odontopediatría. Los criterios de exclusión: las historias clínicas de tratamientos dentoalveolares con datos incompletos.

Se encontró 9880 historias clínicas de pacientes pediátricos que ingresaron al Centro Dental Docente de la UPCH al Servicio de Odontopediatría; de este grupo de historias clínicas, 117 pertenecieron a pacientes que presentaron injurias traumáticas dentales, representando el $1.18 \%$ de pacientes que acudieron al Centro Dental por presentar traumatismos dentales (tabla 1). De las 117 historias clínicas, fueron excluidas 13 porque no cumplieron con los criterios de selección, obteniendo 104 historias clínicas para el estudio. De las 104 historias clínicas de pacientes con injurias traumáticas dentales, 73 fueron de pacientes del sexo masculino y 31 pacientes del sexo femenino

Tabla 1. Injurias traumáticas dentales según la clasificación de Andreasean. Servicio de Odontopediatría - Centro Dental Docente de la Universidad Peruana Cayetano Heredia. Lima-Perú, Enero 2012 - Octubre 2017.

\begin{tabular}{|c|c|c|c|c|}
\hline \multirow{2}{*}{ Variables } & \multicolumn{2}{|c|}{ Dentición Primaria } & \multicolumn{2}{|c|}{ Dentición Permanente } \\
\hline & $\mathbf{n}$ & $\%$ & $\mathbf{n}$ & $\%$ \\
\hline \multicolumn{5}{|l|}{ Injurias traumáticas dentales } \\
\hline Lesiones de los tejidos duros y la pulpa & 49 & 24,87 & 18 & 29,51 \\
\hline Lesiones de los tejidos periodontales & 75 & 38,07 & 20 & 32,79 \\
\hline Lesiones de los tejidos del hueso de sostén & 1 & 0,51 & 1 & 1,64 \\
\hline Lesiones de la encía y/o mucosa & 72 & 36,55 & 22 & 36,07 \\
\hline \multicolumn{5}{|l|}{ Lesiones de los tejidos duros y la pulpa } \\
\hline Fractura del esmalte & 1 & 0,51 & 5 & 8,2 \\
\hline Fractura de esmalte y dentina & 7 & 3,55 & 10 & 16,39 \\
\hline Fractura complicada de corona & 15 & 7,61 & 1 & 1,64 \\
\hline Fractura complicada de corona y raíz & 12 & 6,09 & 0 & 0 \\
\hline Fractura de raíz & 14 & 7,11 & 1 & 1,64 \\
\hline \multicolumn{5}{|l|}{ Lesiones de los tejidos periodontales } \\
\hline Concusión & 23 & 11,68 & 7 & 11,48 \\
\hline Subluxación & 20 & 10,15 & 6 & 9,84 \\
\hline Luxación extrusiva & 2 & 1,02 & 3 & 4,92 \\
\hline Luxación lateral & 11 & 5,58 & 1 & 1,64 \\
\hline Luxación intrusiva & 12 & 6,09 & 1 & 1,64 \\
\hline Avulsión & 7 & 3,55 & 3 & 4,92 \\
\hline \multicolumn{5}{|l|}{ Lesiones de los tejidos del hueso de sostén } \\
\hline Fractura de la mandíbula y maxilar & 1 & 0,51 & 0 & 0 \\
\hline Fractura de la pared alveolar & 0 & 0 & 1 & 1,64 \\
\hline \multicolumn{5}{|l|}{ Lesiones de la encía y/o mucosa } \\
\hline Contusión & 44 & 16,75 & 9 & 14,75 \\
\hline Abrasión & 17 & 8,63 & 5 & 8,2 \\
\hline Laceración & 22 & 11,17 & 8 & 13,11 \\
\hline
\end{tabular}


Tabla 2. Número de injurias traumáticas dentales según sexo, causa del trauma y lugar del accidente acontecido. Servicio de Odontopediatría - Centro Dental Docente de la Universidad Peruana Cayetano Heredia. Lima-Perú, Enero 2012 - Octubre 2017.

\begin{tabular}{|c|c|c|c|c|}
\hline \multirow{2}{*}{ Covariables } & \multicolumn{2}{|c|}{ Dentición Primaria } & \multicolumn{2}{|c|}{ Dentición Permanente } \\
\hline & $\bar{n}$ & $\%$ & $\mathrm{n}$ & $\%$ \\
\hline \multicolumn{5}{|l|}{ Sexo } \\
\hline Femenino & 52 & 26,4 & 32 & 52,46 \\
\hline Masculino & 145 & 73,6 & 29 & 47,54 \\
\hline \multicolumn{5}{|l|}{ Causa del Trauma } \\
\hline Caídas & 87 & 44,16 & 20 & 32,79 \\
\hline Juegos & 106 & 53,81 & 32 & 52,46 \\
\hline Actividad Deportiva & 0 & 0 & 3 & 4,92 \\
\hline Accidente de Tránsito & 4 & 2,03 & 6 & 9,84 \\
\hline Violencia & 0 & 0 & 0 & 0 \\
\hline Otros & 0 & 0 & 0 & 0 \\
\hline \multicolumn{5}{|l|}{ Lugar } \\
\hline Casa & 123 & 62,44 & 8 & 13,11 \\
\hline Alrededor de la casa & 8 & 4,06 & 6 & 9,84 \\
\hline Calle & 20 & 10,15 & 12 & 19,67 \\
\hline Nido/Colegio & 27 & 13,71 & 25 & 40,98 \\
\hline Parque & 12 & 6,09 & 6 & 9,84 \\
\hline Otros & 7 & 3,55 & 4 & 6,56 \\
\hline
\end{tabular}

Tabla 3. Injurias traumáticas dentales según edad (años). Servicio de Odontopediatría - Centro Dental Docente de la Universidad Peruana Cayetano Heredia. Lima-Perú, Enero 2012 - Octubre 2017.

\begin{tabular}{ccccccc}
\hline & \multicolumn{2}{c}{ Dentición Primaria } & & \multicolumn{2}{c}{ Dentición Permanente } \\
\cline { 2 - 3 } Edad (años) & $\mathbf{n}$ & $\mathbf{\%}$ & Edad (años) & $\mathbf{n}$ & $\mathbf{\%}$ \\
\hline 1 & 48 & 24,37 & 7 & 20 & 32,79 \\
2 & 59 & 29,95 & 8 & 3 & 4,92 \\
3 & 27 & 13,71 & 9 & 14 & 22,95 \\
4 & 40 & 20,3 & 10 & 3 & 4,92 \\
5 & 8 & 4,06 & 11 & 6 & 9,84 \\
6 & 11 & 5,58 & 12 & 6 & 9,84 \\
7 & 3 & 1,52 & 13 & 4 & 6,56 \\
8 & 1 & 0,51 & 14 & 2 & 3,28 \\
& & & 15 & 3 & 4,92 \\
\hline
\end{tabular}

(tabla 2), teniendo una edad media de 4,36 $\pm 3,42$ años de edad.

Las variables evaluadas fueron: injurias traumáticas dentales según la clasificación de Andreasean (lesiones de los tejidos duros y la pulpa, lesiones de los tejidos periodontales, lesiones del hueso de sostén, lesiones de la encía o de la mucosa); y las covariables: edad, sexo, causa y lugar de la injuria, y pieza dentaria más afectada.

Los datos fueron obtenidos de la historia clínica de traumatismos dentoalveolares del Servicio de Odontopediatría. Se registraron en una ficha de 
Tabla 4. Injurias traumáticas dentales según tipo de pieza afectada. Servicio de Odontopediatría

- Centro Dental Docente de la Universidad Peruana Cayetano Heredia, Lima-Perú, Enero 2012

- Octubre 2017.

\begin{tabular}{cccccc}
\hline $\begin{array}{c}\text { Pieza dentaria } \\
\text { más afectada }\end{array}$ & \multicolumn{2}{c}{ Dentición Primaria } & $\begin{array}{c}\text { Pieza dentaria } \\
\text { más afectada }\end{array}$ & \multicolumn{2}{c}{$\begin{array}{c}\text { Dentición Permanente } \\
\mathbf{n}\end{array}$} \\
\hline 51 & 134 & 32,52 & 11 & 52 & $\mathbf{\%}$ \\
52 & 29 & 7,04 & 12 & 22 & 14,57 \\
61 & 150 & 36,41 & 13 & 4 & 2,65 \\
62 & 49 & 11,89 & 21 & 50 & 33,11 \\
71 & 9 & 2,18 & 22 & 17 & 11,26 \\
82 & 14 & 3,4 & 31 & 2 & 1,32 \\
63 & 8 & 1,94 & 41 & 4 & 2,65 \\
72 & 6 & 1,46 & & & \\
81 & 9 & 2,18 & & & \\
\hline
\end{tabular}

recolección de datos, diseñada para tal efecto. Se elaboró una base de datos en el programa Excel 2010 y el análisis estadístico descriptivo fue realizado empleando el programa estadístico SPSS 24.0. Los resultados fueron presentados en frecuencias absolutas y relativas mediante tablas descriptivas de doble entrada.

Se obtuvieron los permisos respectivos del Comité Institucional de Ética en Investigación y de las instancias correspondientes de la UPCH, para la ejecución de la investigación.

\section{RESULTADOS}

Se encontró 73 pacientes del sexo masculino y 31 del sexo femenino, encontrándose una edad media de $4,36 \pm 3,42$ años de edad.

En la dentición primaria se encontraron $26,4 \%$ lesiones $(n=52)$ en el sexo femenino y en el sexo masculino $73,6 \% \quad(n=145)$. La frecuencia de traumatismos dentales en dentición primaria según la clasificación de Andreasean fue: a) lesiones de los tejidos periodontales $38,07 \%(n=75)$, b) lesiones de la encía y/o mucosa 36,55\% (n=72), c) lesiones de los tejidos duros y la pulpa dental $24,87 \%(n=49)$ y d) lesiones del hueso de sostén $0,51 \%(\mathrm{n}=1)$. Mientras que en la dentición permanente se encontró: a) lesiones de la encía y/o mucosa $36,07 \%(n=22)$, b) lesiones de los tejidos periodontales $32,79 \%(n=20)$, c) lesiones de los tejidos duros y la pulpa dental 29,51\% $(n=18)$ y d) lesiones del hueso de sostén 1,64\% $(n=1)$. La lesión más frecuente de los tejidos duros y de la pulpa dental fue la fractura complicada de corona 7,61\% $(n=15)$ en la dentición primaria; mientras que la fractura de esmalte y dentina lo fue en la dentición permanente con el 16,39\% $(n=10)$. Las lesiones más frecuentes de los tejidos periodontales fueron la concusión y subluxación en ambas denticiones. Las proporciones de las injurias traumáticas en los tejidos de sostén, fueron bastantes bajas, encontrándose en la dentición decidua: fractura de la mandíbula y maxilar en un $0,51 \%(\mathrm{n}=1)$ y en la dentición permanente: fractura de la pared alveolar en 1,64\% $(n=1)$. La contusión fue la injuria traumática de los tejidos de encía y/o mucosa más frecuente en ambas denticiones (tabla 1). Se observó que la mayor frecuencia de traumatismos dentales en la dentición primaria fue en pacientes masculinos $73,6 \% \quad(n=145)$ mientras que en la dentición permanente fue en el sexo femenino $52,46 \%(n=32)$. La causa más frecuente de dichas lesiones fueron los juegos con un porcentaje de $53,81 \%(\mathrm{n}=106)$ para la dentición primaria y $52,46 \%$ $(n=32)$ para la dentición permanente. El lugar donde sucedió con mayor frecuencia las injurias traumáticas dentales en la dentición primaria fue la casa $62,44 \%$ $(\mathrm{n}=123)$; mientras que, en la dentición permanente, fue en el colegio 40,98\% (n=25) (tabla 2).

En la dentición primaria la mayor prevalencia de injurias traumáticas dentales se encontró en pacientes de 2 años de edad: 29,95\% ( $n=59)$, y en dentición permanente en niños de 7 años de edad: 32,79\% $(n=20)$ (tabla 3). Las piezas dentarias más afectadas fueron los incisivos centrales superiores en ambos tipos de denticiones (tabla 4). 


\section{DISCUSIÓN}

La Organización Mundial de la Salud define la salud como un estado de completo bienestar físico, mental y social. Además, menciona cuatro componentes de la salud: a) estado de adaptación, b) estado fisiológico de equilibrio, c) equilibrio entre la forma y la función del organismo y d) la perspectiva biológica y social (19). La alteración de uno de ellos genera el desequilibrio de la triada entre huésped (sujeto), agente (síndrome) y ambiente (factores que intervienen), generando el estado de enfermedad de la persona (19). Varios autores mencionan que las injurias traumáticas dentales son un problema de salud pública $(5,9,14,15,16,17,20)$, de allí la importancia de medidas que se puedan tomar en los organismos gestionados por el Estado (19).

El análisis de los datos se basó en la verificación de las historias clínicas de injurias traumáticas dentales del Servicio de Odontopediatría del Centro Dental Docente de la UPCH en el periodo de enero 2012 a octubre 2017, siendo la principal razón para elegir este tema la falta de trabajos de investigación sobre lesiones traumáticas dentales en nuestro medio. Sin embargo, debemos mencionar que una de las limitaciones de nuestro estudio es que los pacientes podrían tener como primera opción la atención en el Hospital Cayetano Heredia ya que es un centro de atención de urgencias y emergencias para pacientes que cuentan con el Seguro Integral de Salud (SIS).

De acuerdo con los resultados del presente estudio, los pacientes del sexo masculino fue el grupo más afectado en la dentición primaria, coincidiendo con otros reportes $(12,21,22,23)$. Souza et al., refieren que no encontraron diferencia significativa en cuanto a sexo (24). Oyedele et al., (2) y Gupta et al., (3) nos dicen que esto se debería a que las niñas son más restrictivas en su comportamiento que los niños; ellos tienden a ser más enérgicos y a participar en actividades físicas al aire libre sin protección adecuada y en juegos agresivos. Por otro lado, en la dentición permanente, las niñas fueron las más afectadas con injurias traumáticas dentales, contrario a otros estudios donde refieren que el sexo masculino es el más afectado $(1,2,3,4,7,8,14,23)$. La explicación de lo encontrado en el presente estudio se sustenta en que hay un incremento en la participación de las niñas en deportes o actividades anteriormente practicadas casi exclusivamente por niños $(3,25)$.

Los juegos (grupales o individuales), seguido de las caídas fueron las causas más frecuentes de los traumatismos dentales en ambas denticiones, en el presente estudio; similar a lo encontrado por Ogordi et al., (16); sin embargo, otros autores $(1,3,4,7,8,14,17,18,20,23)$ concluyeron que las caídas fueron las principales causas de traumatismos dentales; además de las colisiones y actividades deportivas, entre otras. Al respecto, Tumen et al., afirman que las caídas son la principal causa de injurias traumáticas dentales debido a la coordinación motora en desarrollo en niños de 5 a 72 meses de edad (22).

En la presente investigación se encontró que el hogar/casa fue el lugar más frecuente donde ocurrieron los traumatismos en la dentición primaria; lo cual corrobora los hallazgos de estudios previos $(17,18,21)$. En la dentición permanente los resultados del presente estudio coinciden con los resultados de Castro et al., al mencionar al colegio como el lugar más frecuente (23), habiendo diferencias con los estudio de Mittals et al., (1), Gupta et al., (3) y Al-Bajjali et al., (7), quienes encontraron al hogar como el lugar de mayor prevalencia $(30,9 \%)$, seguido de la calle $(13,3 \%)$. La explicación de estos resultados se debe a que la mayoría de los niños pasan aproximadamente el $60 \%$ de su tiempo en la casa y el resto en el colegio (3).

Los incisivos centrales superiores seguidos de los incisivos laterales superiores, indistintamente del lado afectado fueron las piezas dentarias más afectadas por traumatismos en ambas denticiones, al igual que la mayoría de estudios consultados (2, $3,4,7,8,10,11,12,13,14,15,16,17,18,22,24,26,27)$. E1 maxilar es un hueso fijo en el cráneo, esto lo hace rígido y aumenta el impacto de las fuerzas dirigida a la cara sobre todo en los dientes antero superiores; mientras que la mandíbula es una parte flexible lo cual le da la ventaja de reducir las fuerzas del impacto en la zona antero inferior mediante el movimiento (22). Además, la erupción temprana, protrusividad, morfología y la posición prominente de los incisivos centrales superiores los hace más susceptibles a traumatismos dentales $(3,7,10,12,13,20)$. 
El presente estudio encontró que la injuria más frecuente en dentición primaria según la clasificación de Andreasean fue la contusión; encontrándose diferencia con estudios previos que nos señalan que la fractura de esmalte fue la lesión traumática más frecuente en la dentición primaria $(11,12,13,18)$. La literatura nos refiere que la subluxación, contusión e intrusión son las lesiones más frecuentes debido a la flexibilidad del hueso alveolar y ligamento periodontal de los dientes primarios (24). Sin embargo, en la dentición permanente, se encontró que la fractura de esmalte y dentina fue la injurias traumáticas dental de mayor frecuencia, igual a lo encontrado por Castro et al., (23). A diferencia de Al-Bajjali et al., (7) y Gupta et al., (3) quienes mencionaron a la fractura del esmalte como la principal lesión en traumatismos dentales.

Las injurias traumáticas dentales más frecuentes fueron las lesiones periodontales seguidas de las lesiones de la encía y/o mucosa. Los niños fueron los más afectados en la dentición primaria y las niñas en la dentición permanente. La mayoría de las lesiones traumáticas dentales se produjeron durante los juegos. La casa y el colegio fueron los lugares donde se produjo la mayor parte de los traumatismos en la dentición primaria y permanente, respectivamente. Los incisivos centrales superiores fueron las piezas dentales más afectadas en este grupo de niños. Se recomienda realizar estudios que involucren factores predisponentes de lesiones traumáticas.

Conflicto de intereses: los autores no tienen conflicto de interés con este informe.

Aprobación de ética: Se solicitó la evaluación y aprobación del Comité Institucional de Ética de la Universidad Peruana Cayetano Heredia para la ejecución del proyecto de investigación. Asimismo, se contó con la autorización del jefe del post-grado de Odontopediatría y la autorización del administrador del Centro Dental Docente de la Universidad Peruana Cayetano Heredia.

Financiamiento: Ninguno.

Contribuciones de los autores: todos los autores contribuyeron en la elaboración de este manuscrito.

Agradecimientos: Agradecimiento a la Mg. Esp. Denisse Jackeline Asián Nomberto, por su apoyo durante el desarrollo del presente trabajo de investigación.

\section{Correspondencia:}

Sandra Alvarez

Jirón Diego de Almagro 587, Jesús María

Lima, Perú

Correo electrónico: sandra.alvarez.r@upch.pe

\section{REFERENCIAS BIBLIOGRÁFICAS}

1. Mittal S, Dogra M, Mittal K, Pathania V, Kaur J. Prevalence of traumatic dental injuries and its correlates among school going children between 8-12 years of age in Sundernager, Himachal Pradesh - a short study. Indian J Dent. 2015; 7(2):5-8.

2. Oyedele T, Jegede A, Folayan M. Prevalence and family structures related factors associated with crown trauma in school children resident in suburban Nigeria. BMC Oral Health. 2016; 16(1):1-7.

3. Gupta M, Kumar S, Kaur J, Gupta S, Bansal V, Dwiedi A. Prevalence, risk factors, and treatment needs of traumatic dental injuries of anterior teeth among 1115 years old children attending government and private schools of Bhopal city, India. JoAOR. 2016; 7(2):32-9.

4. Tümen EC, Yavuz I, Kayd S, et al. Prevalence of traumatic dental injuries and associated factors among 8 to 12 -years-old schoolchildren in Diyarbakir, Turkey. Niger J Clin Pract. 2017; 20(10):1259-66.

5. World Health Organization. Fact sheets. Ginebra: World Health Organization; 2018. ( Citado el 25 de junio del 2020) Disponible en: http://www.who.int/ mediacentre/factsheets/fs318/en/

6. Petti S, Glendor U, Andersson L. World traumatic dental injury prevalence and incidence, a metaanalysis-One billion living people have had traumatic dental injuries. Dent Traumatol. 2018; 34(2):71-86.

7. Al-Bajjali TT, Rajab LD. Traumatic dental injuries among 12-year-old Jordanian schoolchildren: an investigation on obesity and other risk factors. BMC Oral Health. 2014; 14:1-7.

8. Zengin AZ, Sumer AP, Cankaya S. Evaluation of traumatic dental injuries in a group of Turkish population. Niger J Clin Pract. 2015; 18(1):86-9.

9. Aldrigui JM, Jabbar NS, Bonecker M, Braga MM, Wanderley MT. Trends and associated factors in prevalence of dental trauma in Latin America and Caribbean: a systematic review and meta-analysis. Community Dent Oral Epidemiol. 2014; 42(1):30-42.

10. Agostini BA, Pinto LT, Koehler M, Emmanuelli B, Piovesan C, Ardenghi TM. Trend of traumatic crown injuries and associated factors in preschool children. Braz Oral Res. 2016; 30(1):1-8.

11. Berti GO, Hesse D, Bonifácio CC, Raggio DP, Bönecker MJ. Epidemiological study of traumatic dental injuries in 5- to 6-year-old Brazilian children. Braz Oral Res. 2015; 29(1):1-6. 
12. Felix C, Shitsuka C, Moriyama C, Duarte D. Exploratory study of the prevalence of traumatic injuries in preschool children in the city of Macapá, Brazil. Brazilian Research in Pediatric Dentistry and Integrated Clinic. 2014; 14(2):71-77.

13. Piovesan C, Guedes RS, Casagrande L, Ardenghi TM. Socioeconomic and clinical factors associated with traumatic dental injuries in Brazilian preschool children. Braz Oral Res. 2012, 26(5):464-70.

14. Paiva PC, De Paiva HN, De Oliveira Filho PM, Côrtes MI. Prevalence and risk factors associated with traumatic dental injury among 12-year-old schoolchildren in Montes Claros, MG, Brazil. Cien Saude Colet. 2015; 20(4):1225- 33.

15. Dascălu IT, Manolea HO, Coleş E, et al. The prevalence of crown injuries to frontal teeth at schoolchildren aged 6 to 14 and their effects on the periodontal tissue. Rom J Morphol Embryol. 2016; 57:729-35.

16. Ogordi PU, Ize-Iyamu IN, Adeniyi EO. Prevalence of traumatic dental injury to the anterior teeth in children attending paramilitary and nonparamilitary schools in Nigeria. Ann Afr Med. 2019; 18(2):80-85.

17. Siqueira MB, Gomes MC, Oliveira AC, Martins CC, Granville-Garcia AF, Paiva SM. Predisposing factors for traumatic dental injury in primary teeth and seeking of post-trauma care. Braz Dent J. 2013; 24(6): 647-54.

18. Jorge KO, Moysés SJ, Ferreira E, Ramos-Jorge ML, de-Araújo-Zarzar PM. Prevalence and factors associated to dental trauma in infants 1 - 3 years of age. Dent Traumatol. 2009; 25(2):185-89.

19. Editorial Etecé. "Salud (según la OMS)". Enciclopedia de Conceptos; 2017. (Citado el 25 de junio del 2020) Disponible en: http:/concepto.de/salud-segun-la-oms/

20. Guedes OA, de Alencar AH, Lopes LG, Pécora JD, Estrela C. A retrospective study of traumatic dental injuries in a Brazilian Dental Urgency Service. Braz Dent J. 2010; 21(2):153-57.
21. Casals Y, Montero M, González G. Prevalencia de secuelas de traumatismos en dientes temporales en niños institucionalizados del municipio Playa. Rev Cubana Estomatol. 2011; 48(1):14-21.

22. Tumen E, Adiguzel O, Kaya S, Uysal E, Yavuz I, Atakul F. The prevalence and etiology of dental trauma among 5-72 months preschool children in SouthEastern Anatolia Tukey. JIDMR. 2009; 2(2): 40-44.

23. Castro PF, Dreyer E. Prevalencia de traumatismos dentoalveolares en pacientes infantiles del complejo asistencial Dr. Sótero del Río. Rev. Clin. Periodoncia Implantol. Rehabil. Oral. 2012; 5(3): 128-31.

24. Souza M, Silva M, Dos Reis R, De Mota M, De Deus L. Prevalência de traumatismo dentário em préescolares de Teresina, PI. Arq Odontol, Belo Horizonte 2011; 47(1): 18-24.

25. Agbor AM, Nossi AF, Azodo CC, Kamga CL, Zing S. Lesiones maxilofaciales relacionadas con el entrenamiento en el ejército de Camerún. SRM J Res Dent Sci. 2016; 7: 6-9.

26. Tewari N, Mathur VP, Siddiqui I, Morankar R, Verma AR, Pandey RM. Prevalence of traumatic dental injuries in India: A systematic review and metaanalysis. Indian J Dent Res. 2020; 31(4):601-614.

27. Vuletić M, Škaričić J, Batinjan G, Trampuš Z, Čuković Bagić I, Jurić H. A retrospective study on traumatic dental and soft-tissue injuries in preschool children in Zagreb, Croatia. Bosn J Basic Med Sci. 2014;14(1):1225. 